\title{
Deforming mesh with unsteady turbulence model for fluid-structure interaction
}

\author{
J.-T. Yeh \\ Materials Research Laboratories, \\ Industrial Technology Research Institute, Taiwan
}

\begin{abstract}
By taking the mesh of the fluid domain as a virtual solid and using the explicit integration scheme to solve the solid dynamics, a deforming mesh method is proposed for the simulation of fluid-structure interaction. The deforming mesh method with an unsteady turbulence model has been implemented into a finite element code derived from slightly compressible flow formulation and an explicit integration scheme. Due to the explicit integration scheme used for the dynamics of both deforming mesh and fluid flow, it is easy to perform parallel computation for a large-scale fluid-structure interaction problem. After the validation of this approach on the flow induced vibration of the flow past a circular cylinder, the unsteady fluid-structure interaction of a heat exchangertube row in crossflow is demonstrated.

Keywords: deforming mesh, unsteady turbulence model, fluid-structure interaction.
\end{abstract}

\section{Introduction}

The fluid-structure interaction, the unsteady fluid flow making a structure move or deform and the motion of the structure changing the fluid flow, is an important topic in many engineering fields, for example, the flutter of aircraft's wings and the flow induced vibration of tube bundle in heat exchangers. To have a good numerical simulation of the fluid structure interaction, a sufficiently accurate model to solve the unsteady flow field, especially for turbulent flow, and an efficiency method to update the grid/mesh of fluid domain due to the motion of boundaries are of great concern. 
A turbulent flow must be unsteady. To the knowledge of the author, all the major turbulence models, including the large eddy simulation, are not based on the unsteady flow, i.e., once the strain rate exists, the turbulence is computed even though the flow field is steady or without time variation. For example, the unwanted turbulence is computed on the leading edge of flow past an object. In this paper, an unsteady turbulence model where the turbulence is generated from the computed unsteady flow field is presented.

On updating the mesh with moving boundary, re-generation of the mesh with respect to some specified geometric parameters is popularly adopted [1-3]; however, difficulty is encountered when the boundary was not part of a rigid body. A dynamic mesh method [4] with network of artificial springs for the mesh has been developed for the large-scale fluid-structure interactions. However, a large number of linear algebra equations are formed by the implicit time integration scheme of the fluid dynamics or the static analysis of the spring network deformation, and they prove costly when solved using parallel computation. By taking the mesh of the fluid domain as a virtual solid and using the explicit integration scheme to solve the solid dynamics, a deforming mesh method is proposed for the simulation of fluid-structure interaction.

\section{Numerical methods}

In an unsteady flow such as a flow-induced vibration or noise, the propagation of pressure wave exists and the sound speed or the compressibility of the fluid must be considered. The present numerical method for fluid-structure interaction is based on a slightly compressible flow formulation. The continuity equation can be written, instead of the density, in terms of the pressure as

$$
\frac{\partial p}{\partial t}+K u_{i, i}=0
$$

where $p$ is pressure and $u_{i}$ represents the velocity vector. $K\left(=\rho C^{2}\right)$ is the bulk modulus of fluid elasticity ( $\rho$ and $C$ represent the density and the sound speed of a fluid respectively). For a flow field with low Mach number and small density variation, the bulk modulus can be set as a constant.

By the Arbitrary Lagrangian Eulerian description, the momentum equation of the viscous flow with the moving grid velocity vector $\dot{w}_{i}$ is

$$
\rho \frac{\partial u_{i}}{\partial t}=\left(-\delta_{i j} p+\tau_{i j}\right)_{, j}-\rho\left(u_{j}-\dot{w}_{i}\right) u_{i, j}
$$

where $\tau_{i j}\left(=v\left(u_{i, j}+u_{j, i}\right)\right)$ is the shear stress and $v\left(=v_{d}+v_{e}\right)$ represents the total viscosity which is the summation of the dynamic viscosity $v_{d}$ and the effective 
eddy viscosity $v_{e}\left(=\rho l^{2} \gamma\right)$ in the unsteady turbulence model. $l$ is the mixing length, the minimum width of the element or grid. $\gamma$ represents the turbulence strain rate which is generated, dissipated, diffused and convected by

$$
\rho \frac{\partial \gamma}{\partial t}=\rho\left[\dot{\gamma}_{i j}\left|-\frac{\mu+\rho l^{2} \gamma}{\rho C l}\right| \gamma_{i j}|\gamma+| \gamma_{i j} \mid l^{2} \gamma_{, k k}-\left(u_{k}-\dot{w}_{k}\right) \gamma_{, k}\right] .
$$

In the above, $\left|\dot{\gamma}_{i j}\right|$ is the absolute value of the variation rate of the strain rate

$$
\gamma_{i j}=\left(u_{i, j}+u_{j, i}\right) / 2 \text { or }\left|\dot{\gamma}_{i j}\right| \Delta t=\sqrt{2 \Delta \gamma_{i j} \Delta \gamma_{i j}} .
$$

The moving grid is taken as a solid with the same density and bulk modulus of the fluid, and governed by

$$
\rho \frac{\partial^{2} w_{i}}{\partial t^{2}}=\sigma_{i j, j}
$$

where the stress $\sigma_{i j}$ is derived from the displacement field $w_{i}$ by a constitutive equation.

After dividing the computation domain by finite elements, the field variable, velocity $u_{i}$, turbulence strain rate $\gamma$ and moving grid displacement $w_{i}$ can be represented by the interpolation of nodal variable $\{U\},\{\Gamma\}$ and $\{W\}$. Based on the weighted residual method, the weak-form of the equilibrium equations derived from eqns. (2)-(4) and boundary conditions with quadrilateral (2D) or hexahedron (3D) element and reduced integration can be written as

$$
[M]\left\{\frac{\partial U}{\partial t}\right\}=\left\{R_{U}\right\},[M]\left\{\frac{\partial \Gamma}{\partial t}\right\}=\left\{R_{\Gamma}\right\} \text { and }[M]\left\{\frac{\partial^{2} W}{\partial t^{2}}\right\}=\left\{R_{W}\right\}
$$

where $[M]$ is the mass matrix; $\left\{R_{U}\right\},\left\{R_{\Gamma}\right\}$ and $\left\{R_{W}\right\}$ are the corresponding unbalanced or resultant nodal residuals. By using the explicit time integration scheme and the lumped, therefore diagonal, mass matrix, the above equations are efficient to be solved and easy to be implemented into parallel computation for a large scale fluid-structure interaction computation.

It is noticed that the pressure is not a nodal variable but is a derived quantity in an element solved by eqn. (1). Since that the wave propagation distance $(=C \Delta t)$ during a time increment is in usual much less than the element width $l$ and numerical divergence is happened by this numerical scheme, a pressure damping $(=\dot{p}(\sqrt{l / C \Delta t}-1))$ is added in solving the momentum equations. In order to prevent using an element that is too thin, a slipping boundary condition 
is used where the nodes are put on one quarter of element thickness away from the wall and the corresponding shear stresses due to viscosity are applied.

\section{Examples}

Two examples have been carried out to demonstrate the accuracy and versatility of the numerical method for flow induced vibration. One is the typical flow past a single flexible circular cylinder, the other is the tube row in crossflow.

\subsection{The flow past a single flexible circular cylinder}

Figure 1 shows the computation domain of the flow past a cylinder and the local mesh near the cylinder. The Reynolds number of the flow field is 1.E5 while the density, dynamic viscosity, free stream velocity of the fluid and the diameter (D) of the cylinder are given in a consistent unit as 1, 2.E-8, 1 and 2.E-3, respectively. The upstream (left) boundary and the two sides (top and bottom) are $20 \mathrm{D}$ away from the cylinder center. The downstream boundary is extended to $40 \mathrm{D}$.

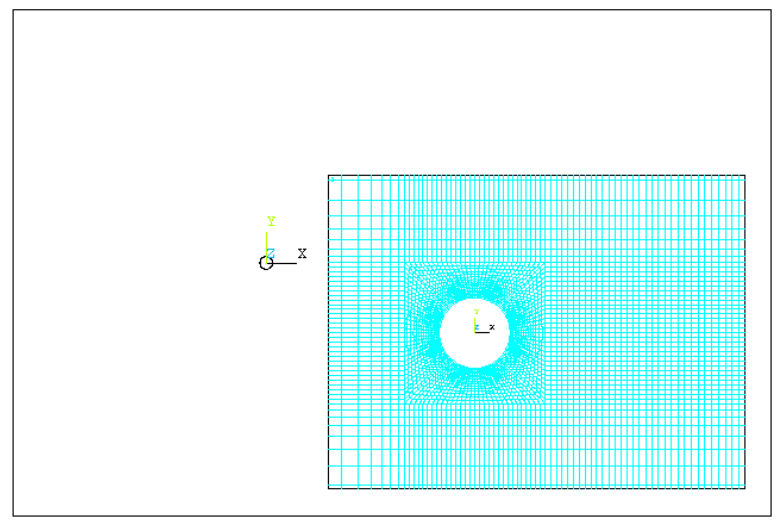

Figure 1: The computation domain and the local mesh near cylinder.

When the cylinder was fixed, the variations of computed drag (X) and lift (Y) forces are plotted in Figure 2. The free stream velocity is risen up during the time from 0 . to 0.01 and so the drag force is. From the lift force, the flow field is symmetric for two vortices and the lift is almost vanished at the beginning. It gradually changes to be non-symmetric or shaking vortex street shown in Figure 3 and the lift force comes up before about Time $=0.15$. After being in vortex shaking, the averaged drag, root mean squared lift and the shaking frequency have a good agreement with experiments [5] and the non-perfect periodic variation shows the chaotic behavior of the turbulent flow. The computed distribution of turbulence strain rate at an instant as displayed in Figure 4 shows quite similar to that taken from shadowgraph in Figure 5 [6]. 


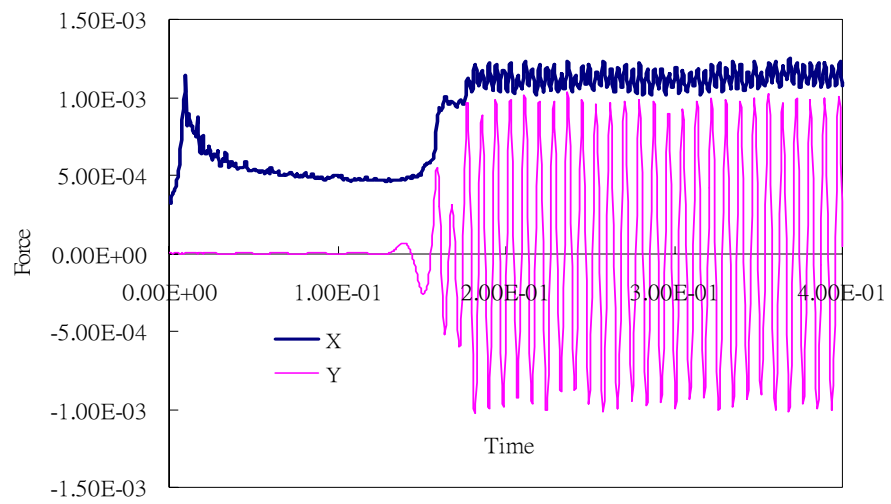

Figure 2: $\quad$ The variations of computed $\operatorname{drag}(\mathrm{X})$ and lift $(\mathrm{Y})$ forces.

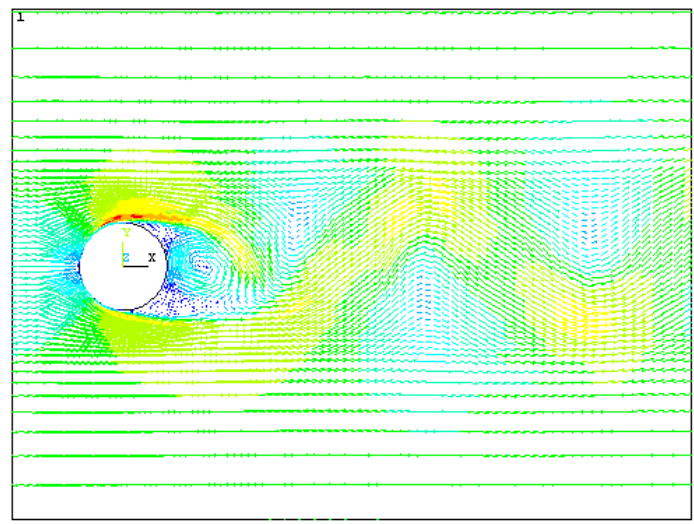

Figure 3: The snapshot of velocity field for the flow past a cylinder.

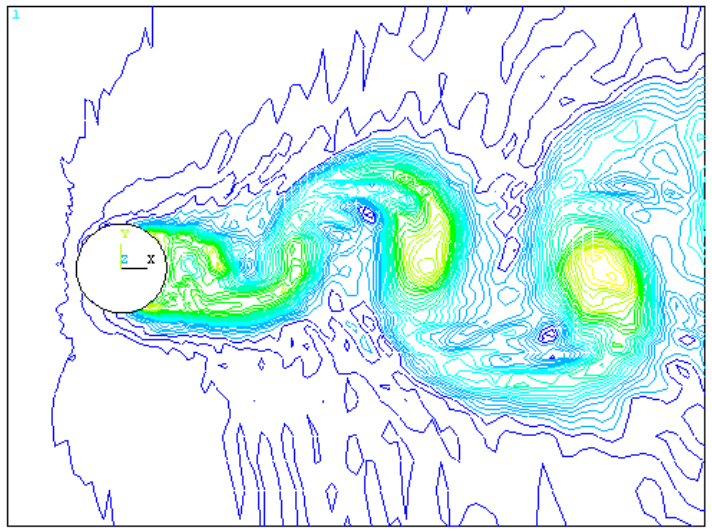

Figure 4: The snapshot of turbulence strain rate. 


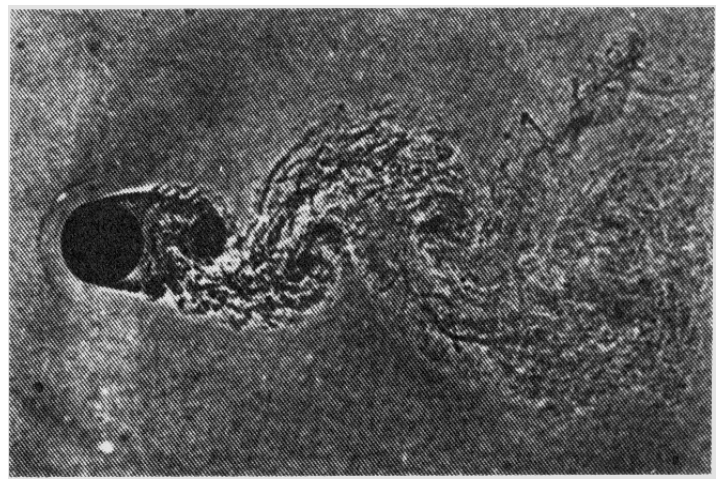

Figure 5: The multiple-spark shadowgraph of turbulence.

When the circular cylinder was flexible, it is supported by a spring and a damper on both $\mathrm{X}$ and $\mathrm{Y}$ directions in this two-dimensional model. In order to show significant flow induced vibration, the resonance frequency of the cylinder is designed to match the shaking frequency of vortex street. Therefore, the mass of the cylinder $(\mathrm{M})$, the spring constant $(\mathrm{K})$ and a small damping $(\mathrm{C})$ are given in a consistent unit as 7.782e-4, 388.8 and 0.00118, respectively. Figure 6 shows the deformed mesh in significant displacement and the nodal velocity at an instant. It is interesting to find that the nodal velocities of mesh are not all inphase due to the deforming mesh method being used. As shown in Figure 7, the computed lateral oscillation begins to be amplified as expected due to the resonance after the vortex shaking occurs. The amplifying lateral oscillation makes the averaged drag force slightly increasing as given in Figure 8 which agrees with the observation in experiments [5].

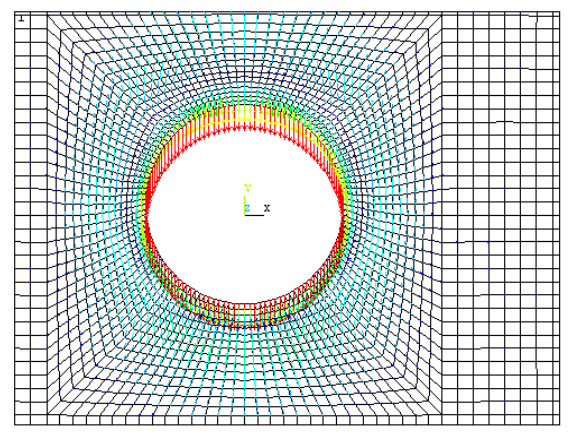

Figure 6: The deformed mesh and the nodal velocities of mesh at an instant.

\subsection{The tube row in crossflow}

Figure 9 shows the computation domain and the local mesh of the tube row in crossflow [5] considered. The diameter of tube is $0.0159 \mathrm{~m}$. The ratio of pitch to 
diameter is 1.35 . In unit length $(\mathrm{m})$, the mass of tube is $3.861 \mathrm{~kg}$, the equivalent spring constant and damping are given as $103.834 \mathrm{~N} / \mathrm{m}$ and $57.14 \mathrm{~kg} / \mathrm{sec}$, respectively in the two-dimensional model. The density of fluid is $1000 \mathrm{~kg} / \mathrm{m}^{3}$ and its dynamic viscosity is $0.001 \mathrm{~N}-\mathrm{sec} / \mathrm{m}^{2}$. A flow rate to have the average velocity $1.5 \mathrm{~m} / \mathrm{sec}$ in the gaps between the tubes is given.

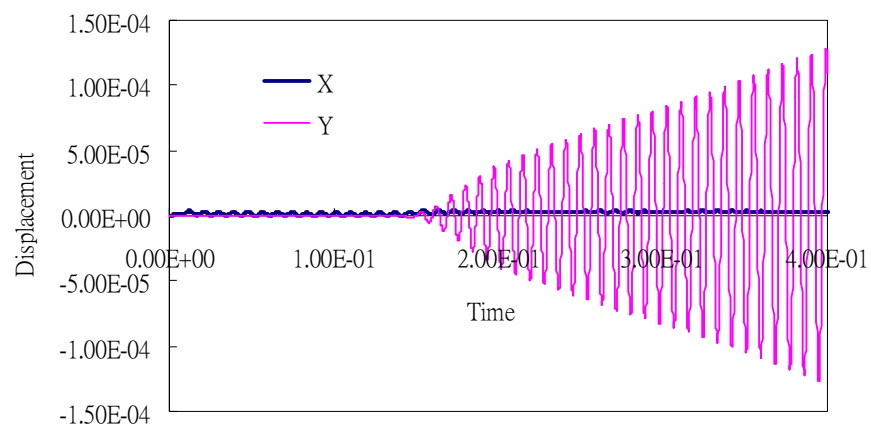

Figure 7: The variations of computed displacements.

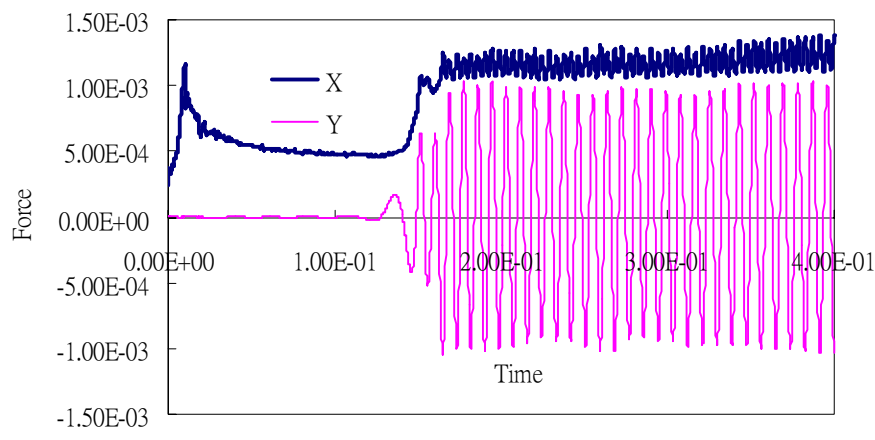

Figure 8: $\quad$ The variations of computed drag $(\mathrm{X})$ and lift $(\mathrm{Y})$ forces.

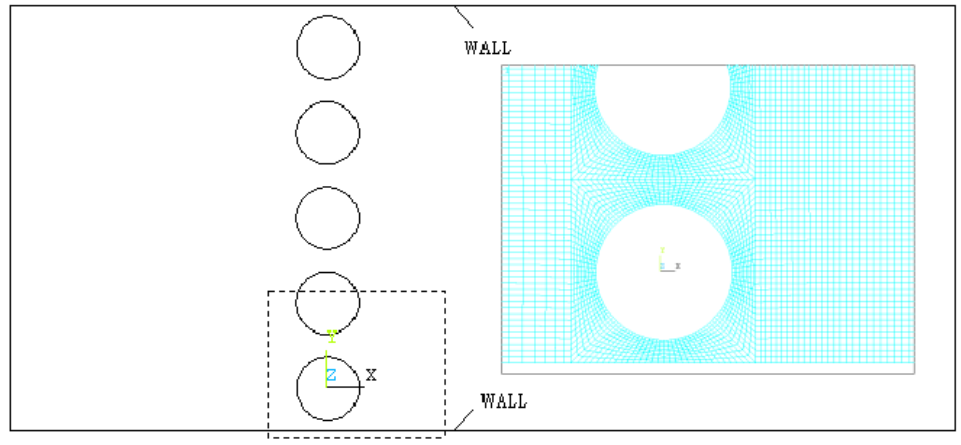

Figure 9: The computation domain of the tube row in crossflow. 
Figure 10 and 11 give the snapshots of velocity field and turbulence strain rate for the tube row in crossflow. The unequal spacing between the tubes shows the large displacement of them at that moment. It seems that the jet flow with less turbulence in the gaps penetrates into the fully developed turbulent zone.

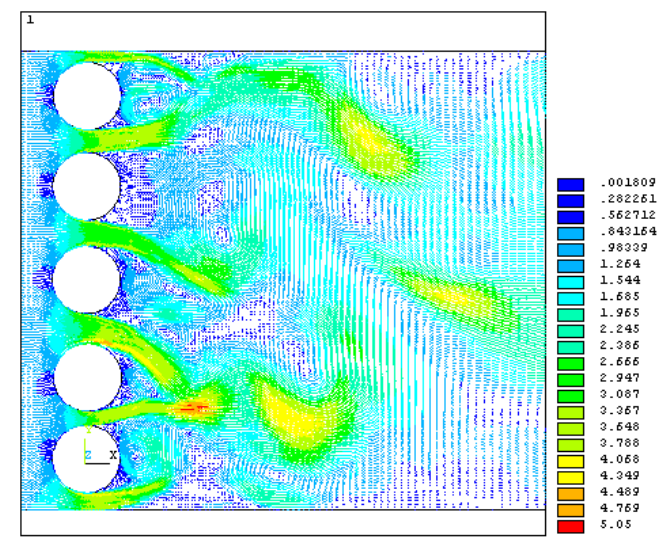

Figure 10: The snapshot of velocity field for the tube row in crossflow.

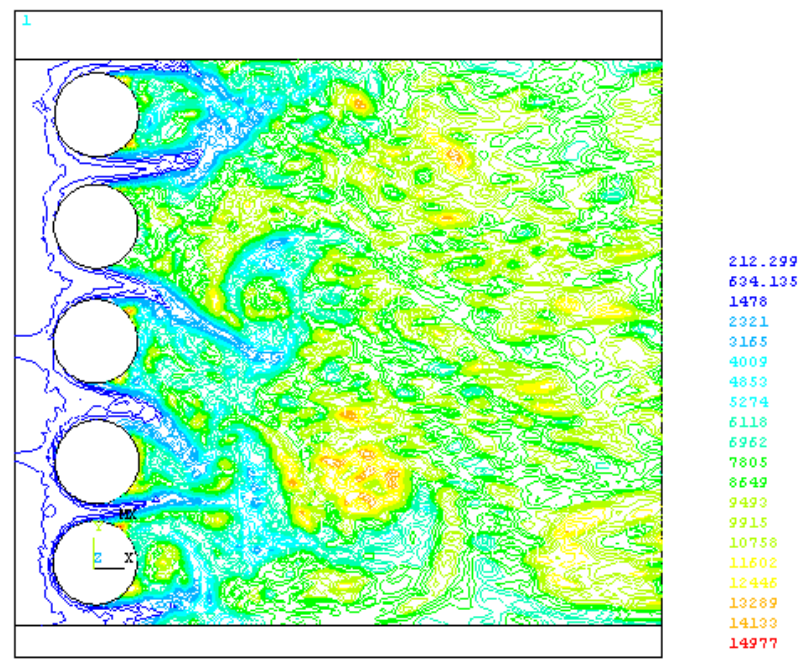

Figure 11: The snapshot of turbulence strain rate for the tube row in crossflow.

Due to a quick start-up of incoming flow from the upstream, all tubes are subjected to an impulsive force and oscillating simultaneously at the beginning as shown in the variation of displacement, Figure 12. The same as the flow past a single cylinder, the symmetry of geometry makes the middle tube (No. 3) show no lateral displacement in the early stage rather than the others. After the vortex 
shaking of the middle tube coming up, it seems that there is no significant difference of the oscillation type between all of the tubes. Although the oscillation in the nature frequency of the tube is observed, more significant chaotic fluctuation induced by the turbulence is noticed.
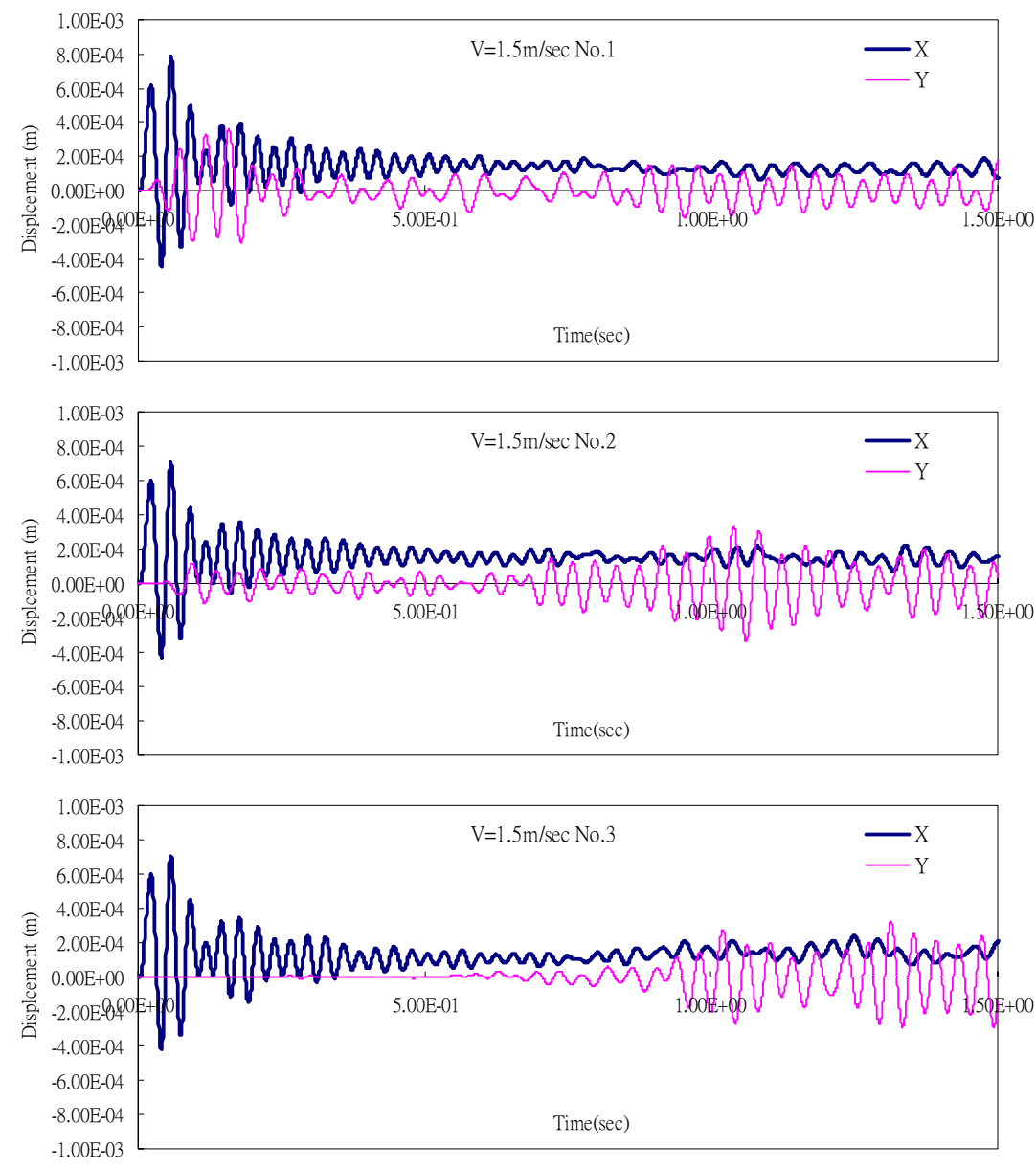

Figure 12: The variations of computed displacements where No. 1 is the tube near the wall, No. 2 is the next and No. 3 is the middle tube.

\section{Concluding remarks}

The proposed deforming mesh with the unsteady turbulence model shows good results on the fluid-structure interaction of the flow past a cylinder or tube row. The present method has been extended to three-dimensional flow fields and the parallel computation. Further development to free surface flow of material processing, such as the die coating, will be reported in the future. 


\section{References}

[1] Schroder, K. and Gelbe, H., Two- and Three- Dimensional CFDSimulation of Flow- Induced Vibration Excitation in Tube Bundles. Chemical Engineering and Processing Vol. 38, pp. 621-629, 1999.

[2] Ichioka, T., etc, Research on Fluid Elastic Vibration of Cylinder Arrays by Computational Fluid Dynamics. JSME International Journal, Series B. Vol. 40, No. 1, pp. 16-24, 1997.

[3] Sadaoka, N., etc, Analysis of Flow- Induced Vibrations in Piping Systems and Circular Cylindrical Structures. JSME International Journal Series B, Vol. 41, No. 1, pp. 221-226, 1998.

[4] Farhat, C., etc, Torsional springs for two-dimensional dynamic unstructured fluid meshes. Computer Methods in Applied Mechanics and Engineering, Vol. 163, No. 1-4, pp. 231-245, 1998.

[5] Chen, S-S. Flow-Induced Vibration of Circular Cylindrical Structures, Hemisphere Pub. Corp., 1987.

[6] Van Dyke, M., An Album of Fluid Motion, The Parabolic Press, 1982. 